\title{
Adhesion and Mechanical Properties of TiAlN and AlTiN Magnetron Sputtered Coatings Deposited on the DMSL Titanium Alloy Substrate
}

\begin{abstract}
M. Walczak, K. Pasierbiewicz, M. Szala*
Lublin University of Technology, Faculty of Mechanical Engineering, Department of Materials Engineering, Nadbystrzycka St. 36, 20-618 Lublin, Poland

Consideration of thin films such as deposition of TiAlN or AlTiN on DMLS (direct metal laser sintered) titanium-based implants is a new biomedical trend. The present study encompassed the investigations of hardness, Young modulus, adhesion of TiAlN and AlTiN magnetron sputtered coatings deposited on DMLS (direct metal laser sintering) of Ti6Al4V substrate. The titanium based substrate was manufactured in the DSLM process by means of 3D EOSINT M280 printer for metals supplied by EOS. The surface morphology of specimens was examined using the Dektak 150 profilometer (Veeco Instruments Inc., USA). The mechanical properties (hardness, Young's modulus) of nitride coatings and the substrate were tested by means of Ultra Nanoindentation Tester (Anton Paar $\mathrm{GmbH}$, Germany). The values of hardness and elastic modulus were calculated from the load-displacement data according to the Oliver Pharr method. The adhesion of deposited coatings was determined by means of the scratch test and Rockwell test. Scratch tests were performed on a Micro Combi Tester (Anton Paar GmbH, Germany) according to the ASTM C1624 - 05(2015) standard for ceramic coatings scratch testing. It was found that TiAlN as well as AlTiN films were characterised by excellent adhesion to DMLS titanium-based substrate as well as sufficient morphology and mechanical properties.
\end{abstract}

DOI: 10.12693/APhysPolA.136.294

PACS/topics: 68.37.-d, 68.35.Np, 71.55.Ak, 81.15.Cd, 89.20.Kk

\section{Introduction}

Consideration of thin films such as deposited TiAlN and AlTiN on DMLS (direct metal laser sintered) titanium-based implants is a new biomedical trend. So far, according to the literature abrasive wear has been one of the most frequent causes of biomaterials damage [1]. This mechanism of wear leads to reduced implant life and an increased number of necessary revision treatments. Abrasive wear products result in the decreased mechanical properties, particularly on the contact of a tribological pair. Moreover, they intensify pain and negative biological response of tissues which leads to local irritation and inflammation and consequently, to implant failure. Titanium and its alloys are very popular in medicine although the cobalt based and nickel based alloys are still applicable as a biomaterials [1-7]. Unfortunately, titanium alloys have insufficient abrasion resistance. In recent years there has been a trend towards modification of the surface layer of titanium materials. This trend, among others is related to the application of nitride coatings using the PVD method such as TiAlN and AlTiN films. PVD coatings enable to obtain a gradient diffusion layer with sufficient thickness and adhesion which ensures a long-lasting abrasionresistant surface. Nitride layers formation technology using the PVD methods is popular in the processes for

*corresponding author; e-mail: m.szala@pollub.pl improving the properties of biomaterials or machining tools. Unfortunately, reports in the literature indicate that the films are usually deposited on the bulk metal substrates $[4,6,8-12]$ or the cemented carbides substrates $[8,13,14]$. Moreover, the literature survey indicates that there are no reports regarding morphology, mechanical properties, and adhesion of coatings of AlTiN and TiAlN deposited on the DMLS titanium-based substrates. Insufficient quality of surface finishing in combination with wear and corrosion processes leads, among others, to premature failure of implants and to revision surgeries.

Therefore, for increasing the wear resistance of titanium substrates the modification of surface layer with deposition of PVD nitride coatings is proposed. Hence the purpose of the present study was to investigate the TiAlN and AlTiN coatings deposited on the Ti-6Al-4V substrate.

DMLS technology is a unique process employed in customized implants manufacturing $[2,4]$. The process fuses metal powder into a solid part by melting it locally using the focused laser beam where it traces the cross section of the object layer by layer. Elements produced in the DMLS process are characterized by visible, particularly at the surface layer, traces produced after fusing metal powder into the solid part by melting it locally using the focused laser beam (Fig. 1). In addition, the products obtained in the DMLS process are expected to demonstrate the presence of residual tensile stresses after sintering [15]. M. Benedetti et al. [16] reported that maximal residual stresses occur at the depth of 0.15 
to $0.2 \mathrm{~mm}$. However, there are no reports in the literature on the adhesion of PVD coatings applied in the DMLS process on the substrate characterized by high residual stresses in the surface layer. As follows from the literature review the data related to the mechanical properties of selective-laser-melting in titanium ( $\mathrm{Ti}-6 \mathrm{Al}-4 \mathrm{~V}$ ) produced in the DMLS process are also neglected while reporting thin films properties $[15,17]$. Therefore its application seems to be rather difficult. As there are known production methods of sputtered films e.g. TiAlN deposited on implants $[3,4]$, it is worth considering the usage of thin films on titanium implants produced in the DMLS process, for example in designing of customized hip joint implants. Analogous investigations are carried on in the scope of potential AlTiN application as a DMLS biomaterial. Additionally, as a result of intensive development in the scope of DMLS technologies in the last decade, it is possible to produce the so called personalized implants. Therefore the present study focuses on examination of applicability of AlTiN and TiAlN coatings on the titanium substrate (Ti6Al4V) produced using the DMLS technology.

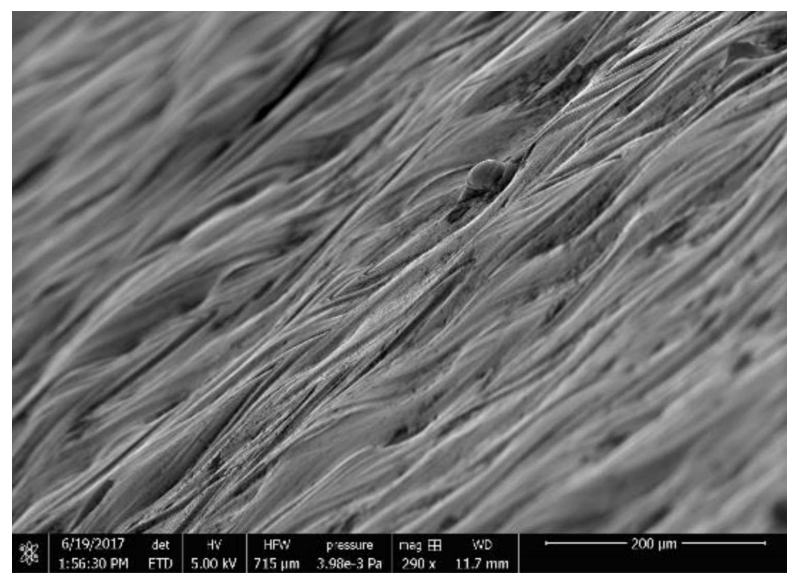

Fig. 1. Metal surface of element produced in DMLS process - own study of the authors.

The aim of this study was to investigate the mechanical properties of sputtered TiAlN and AlTiN films deposited on the DMSL (direct metal laser sintered) titanium-based substrate as well as to provide the mechanical properties of DMLS manufactured Ti-6Al-4V alloy.

\section{Materials and methods}

AlTiN and TiAlN coatings were deposited on the substrate manufactured in the DMLS process. The substrates were made of titanium (Ti6Al4V grade) powder fused by means of 3D EOSINT M280 metal printer (manufactured by EOS GmbH, Germany) with the standard DMLS process procedure parameters specified by the printer manufacturer. Figure 1 illustrates the surface of DMLS titanium-coated specimens. Then, substrates were machined, ground, and polished to obtain the $S_{a}$ parameter of surface roughness on the level of $0.1 \mu \mathrm{m}$. The AlTiN and TiAlN coatings were deposited physically from the gas phase PVD in the magnetron sputtering process. Films differ in chemical composition which was measured using SEM-EDS method on the samples surfaces (Table I). The sputtering process was preceded by preliminary heating to $400^{\circ} \mathrm{C}$, ion etching by means of $100 \mathrm{~V} \mathrm{DC}$ with the current of $20 \mathrm{~A}$, and then by means of modulated current with the frequency of $240 \mathrm{kHz}$. The sputtering process was performed in vacuum below $10^{-7} \mathrm{~Pa}$ in the argon and krypton atmosphere.

Coatings thickness and chemical composi-

TABLE I tion estimated with SEM-EDS.

\begin{tabular}{c|c|c}
\hline \hline Coating & $\begin{array}{c}\text { Coating } \\
\text { thickness }[\mu \mathrm{m}]\end{array}$ & $\begin{array}{c}\text { Content } \\
\text { of chemical } \\
\text { elements [at.\%] }\end{array}$ \\
\hline AlTiN & $4-5$ & $24 \% \mathrm{Ti}, 35 \% \mathrm{Al}, 41 \% \mathrm{~N}$ \\
TiAlN & $4-5$ & $32 \% \mathrm{Ti} ; 31 \% \mathrm{Al} ; 37 \% \mathrm{~N}$
\end{tabular}

The roughness of substrates and coatings were determined by means of the Contour GT profilometer (manufactured by Bruker, Germany). The mechanical properties of nitride coating and the substrate, such as hardness, Young's modulus were determined by means of Ultra Nanoindentation Tester (Anton Paar GmbH, Germany). The 30 indentations were made using the Berkovich diamond indenter with the load $20 \mathrm{mN}$. Accordingly to the literature of the subject $[18,19]$ the indentation depth $(h)$ was always below $10 \%$ of the coating thickness. The hardness and elastic modulus were calculated from the load-displacement data according to the Oliver Pharr method [20]. Therefore, the instrumented elastic modulus $E_{\mathrm{IT}}$ was estimated taking into consideration the Poisson ratios of diamond inventor $\nu_{i}$ and tested film $\nu_{s}$, as well as $E_{r}$ determined by the Oliver Pharr method. $E_{\mathrm{IT}}$ was calculated based on the ISO 14577-1:2015 standard form formula (1):

$$
E_{\mathrm{IT}}=\frac{1-\left(\nu_{s}\right)^{2}}{\frac{1}{E_{r}}-\frac{1-\left(\nu_{s}\right)^{2}}{E_{i}}}
$$

where $E_{\mathrm{IT}}$ is the instrumented elastic modulus, $\nu_{s}$ is the Poisson ratio of the test piece, $\nu_{i}$ is Poisson ratio of the indenter, $E_{r}$ is the reduced modulus of the indentation contact, $E_{i}$ is the modulus of the indenter.

The coating adhesion was determined by means of the scratch test and the Rockwell test. The scratch tests were taken on a Micro Combi Tester (Anton Paar GmbH, Germany) according to the ASTM C1624 standard for ceramic coatings scratch testing, 10 scratches were made for each specimen at the progressive force (start load of $0.05 \mathrm{~N}$ and the end load of $30 \mathrm{~N}$ ) and the rate of $5 \mathrm{~N} / \mathrm{s}$. The Rockwell test was conducted according to the VDI 3198 procedure in order to compare the films adhesion with the substrate. Accordingly to [21, 22] the Rockwell indentation is classified into six ratings evaluating the damage degree from HF1 to HF6. HF1 
to HF4 indicating adequate adhesion, and no evident spallation can be seen around the boundary. In contrast, for HF5 and HF6, severe delamination took place which reflects inadequate adhesion between the film and the substrate. The parameters of test measurements are described in detail in [21, 23].

\section{Results and discussion}

Figure 2 illustrates the structures of AlTiN and TiAlN film surfaces The difference between films relies on its chemical composition (Table I). The AlTiN film was enriched in aluminum Observations conducted using the scanning electron microscope allows to proof that both magnetron sputtered films were fine grained and presented dense structure. In addition, it is known from the literature that the mechanism of film growth by sputtering follows an adatom process that is strongly affected by the substrate orientation (similar to an epitaxial growth). That phenomenon is confirmed and clearly visible on the 3D maps of surface topography, (Fig. 3), where the laser beam sintering traces can be identified in the measurements by means of profilometer. On the whole, the coating films morphology is smooth and uniform, the values of $S_{a}$ parameters of roughness are relatively low i.e. $0.027 \mu \mathrm{m}-0.028 \mu \mathrm{m}$ for the AlTiN and TiAlN coatings. The roughens increases three times in comparison with the substrate roughness, $S_{a}=0.01 \mu \mathrm{m}$.
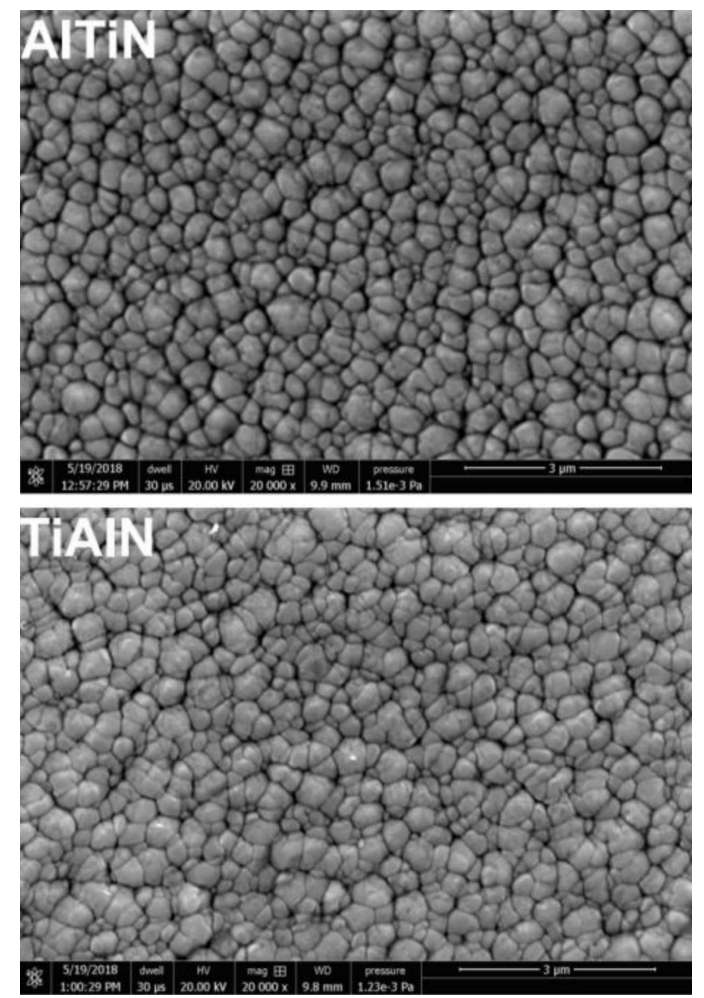

Fig. 2. SEM microphotographs illustratingsurface of PVD coatings.

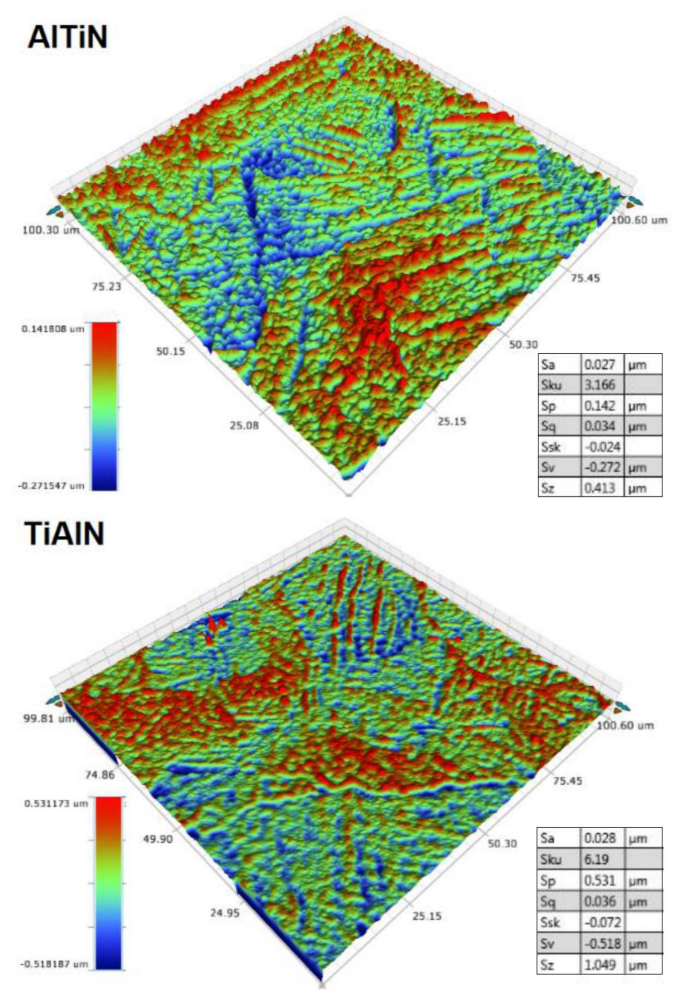

Fig. 3. Morphology and roughness parameters of AlTiN and TiAlN coatings.

The elastic modulus and hardness of the deposited coating and Ti-6Al-4V DMLS produced substrate are summarized in Figs. 4 and 5 . It can be stated that the study of titanium substrate exhibits characteristic properties of plastic material (Fig. 4). Therefore the obtained mechanical properties are comparable to those of Ti-6Al-V4 bulk metal alloy reported by other scientists [2]. On the whole, the laser-produced base demonstrates up to four times lower hardness and Young module than nitride coatings. In addition, the coatings are characterised by superior mechanical properties (elastic modulus and microhardness) in comparison with the substrate. Moreover, TiAlN is characterised by $21 \%$ higher value of elastic modulus as well as $16 \%$ higher value of microhardness than the AlTiN coating. As it can be seen on the average the TiAlN coating is harder and more rigid than the AlTiN coating and the mechanical properties of deposited coatings are comparable with the literature data $[8,23-25]$. Besides that the obtained hardness results of PVD coatings deposited on the DMLS titanium substrate are in the range of those presented for coatings deposited on the bulk Ti-6Al-4V alloy, specified in [23]. Furthermore, according to the literature $[3,25]$, the $H / E$ values (hardness / elastic modulus) can be used as an indicator of the wear initiation. The higher $H / E$ value indicates greater resistance to abrasion with higher hardness prior to smaller amount of residual steress. In the paper, the average $H / E$ values were calculated for the coatings and comparable results were obtained for the other values, $H / E_{\mathrm{AlTiN}}=0.055$ and $H / E_{\mathrm{TiAlN}}=0.053$. 


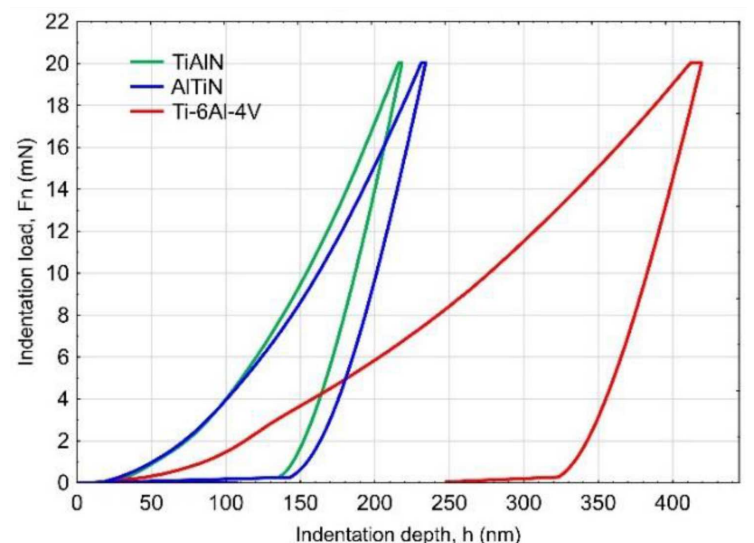

Fig. 4. Loading-unloading nanoindentation curves for tested materials.
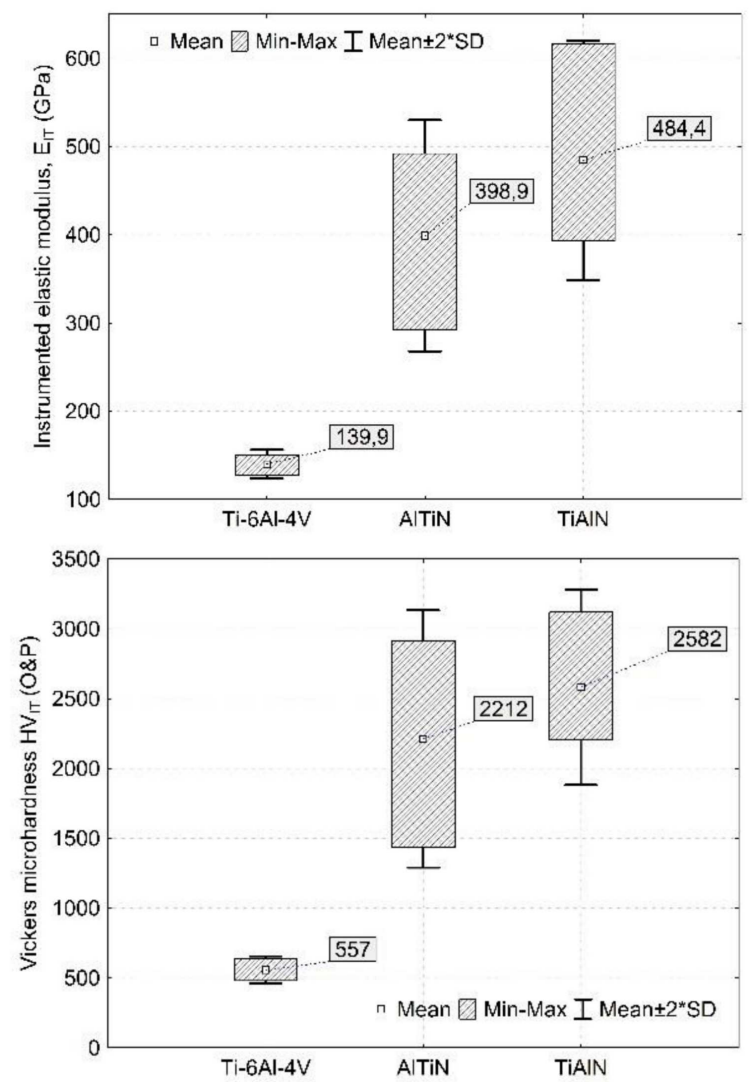

Fig. 5. Mechanical properties obtained in nanointedation testing.

Based on the analysis of Rockwell tests results the acceptable failure of AlTiN and TiAlN coatings to substrates was determined. The adhesion of the both nitride PVD coatings was on the high level of HF1 (according to VDI 3198 procedure) which means the smallest number of cracks was near the indentation boundaries and this implies sufficient films to the substrates binding Fig. 6 shows oval indentations left by the Rockwell indenter and cracks of AlTiN and TiAlN coatings as a result of pressure and deformations. As expected, the highest residual stress is situated in the centre of indentation. In the course of adhesion assessment, particular attention is paid to the area surrounding the indentations characterized by potential occurrence of coating cracks and chipping. For the AlTiN and TiAlN coatings, no chipping of coating was observed and visible cracks were caused by substrate deformation causing cracks within the film of the two tested coatings, the TiAlN seems to be more durable which can be due to the higher values of nanohardness and elastic modulus of the TiAlN than AlTiN coating (Fig. 5). No film delamination was observed even in the extremely deformed areas. The TiAlN and AlTiN coatings adhere to the DMLS substrate very closely.
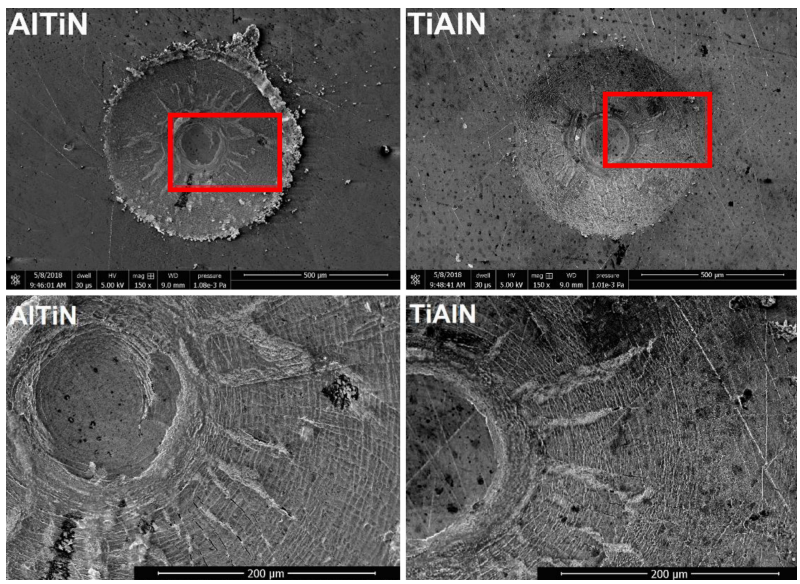

Fig. 6. Adhesion Rockwell tests of PVD thin films deposited on DLMS titanium-substrate, enlarged selected area $(\mathrm{SEM})$.

The examples of scratch testing findings are presented in Fig. 7 and Table II contains the critical loads which are identified as $L_{c 1}, L_{c 2}$, and $L_{c 3}$ for cracking, spallation on the scratch track border, and coating delamination in the centre of the track, respectively. The analysis of scratch test indicates the cohesive wear mechanism visible at the edges of the scratch (chevron cracks) while in the middle of the scratch trace conformal cracking was observed (Fig. 7). Examination of critical loads $\left(L_{c 1}, L_{c 2}\right.$, and $\left.L_{c 3}\right)$ shows lower average values for the AlTiN than TiAlN coating (Table II). However, the results are included in the deviation range. The obtained critical load results are comparable with those reported in the literature [11]. Both the Rockwell and scratch tests indicate proper adhesion of PVD coatings to the DMSL titanium substrate. Therefore it was possible to confirm comparable adhesion of both tested nitride films. Advantageous mechanical properties and morphology of PVD coatings indicate that the AlTiN and TiAlN coatings produced on the DMLS titanium-based substrate can be used in biomedical engineering, particularly in the dedicated implant design. 


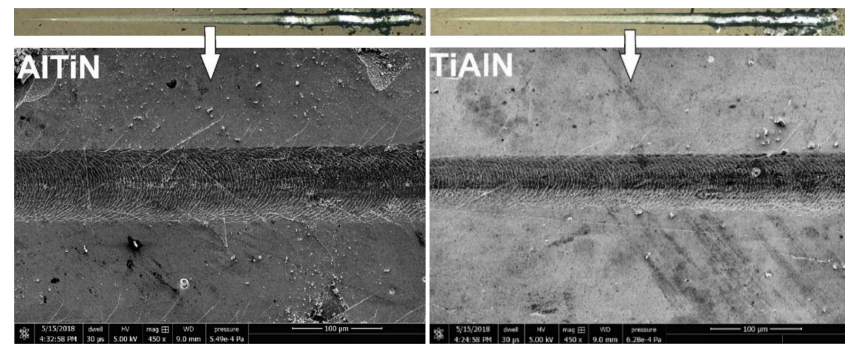

Fig. 7. Scratch traces (total scratch length $3 \mathrm{~mm}$ ) and micrographs of scratch traces (results in Table II).

TABLE II

Results of scratch testing of nitride coatings (critical loads $L_{c 1}, L_{c 2}$ and $L_{c 3}$ )

\begin{tabular}{c|c|c|c}
\hline \hline Coating & $L_{c 1}[\mathrm{~N}]$ & $L_{c 2}[\mathrm{~N}]$ & $L_{c 3}[\mathrm{~N}]$ \\
\hline AlTiN & $2.42 \pm 0.29$ & $19.06 \pm 2.87$ & $25.81 \pm 1.52$ \\
TiAlN & $2.65 \pm 0.29$ & $21.36 \pm 1.49$ & $26.16 \pm 0.81$
\end{tabular}

\section{Conclusions}

The following conclusions can be drawn on the basis of the research.

Morphology and properties of TiAlN and AlTiN coatings deposited by means of the PVD process on the DMLS titanium-based substrate were uniform and reproducible. The coatings exhibit comparable $S_{a}$ values of surface roughness for AlTiN and TiAlN.

Mechanical properties of DMLS substrate and magnetron sputtered films were identified. Coatings are characterized by superior mechanical properties (elastic modulus and microhardness) to the substrate. Moreover, TiAlN is characterised by $21 \%$ higher value of elastic modulus as well as $16 \%$ higher value of microhardness than the AlTiN coating.

The Rockwell and scratch tests evidenced good adhesion of AlTiN and TiAlN films to the titanium substrate manufactured in the DMLS process. PVD coatings adhesion Rockwell test gives acceptable failure results on the highest level of HF1 (excellent adhesion films to the substrates). The analysis of the scratch test results indicate cohesive wear mechanism visible at the edges of the scratch trace (chevron cracks) while in the middle of the scratch trace conformal cracks were observed. This finding confirms proper adhesion of PVD coatings to the DMSL titanium substrate. The examination of critical loads $\left(L_{c 1}, L_{c 2}\right.$, and $L_{c 3}$ ) shows lower values for the AlTiN than TiAlN coating.

The advantageous mechanical properties and morphology of PVD coatings indicate that the AlTiN and TiAlN coatings produced on the DMLS titanium-based substrate can be used in medicine, particularly in individual implant designing.

\section{References}

[1] M. Walczak, K. Drozd, Curr. Issues Pharm. Med. Sci. 29, 158 (2016).

[2] W.S.W. Harun, N.S. Manam, M.S.I.N. Kamariah, S. Sharif, A.H. Zulkifly, I. Ahmad, H. Miura, Powder Technol. 331, 74 (2018).

[3] F. Yıldız, A. Alsaran, A. Çelik, İ. Efeoğlu, Surf. Eng. 26, 578 (2010).

[4] A.L. Jardini, M.A. Larosa, C.A.D.C. Zavaglia, L.F. Bernardes, C.S. Lambert, P. Kharmandayan, D. Calderoni, R.M. Filho, Virtual Phys. Prototyp. 9, 115 (2014).

[5] R. Karpiński, Ł. Jaworski, M. Szala, M. Mańko, ITM Web Conf. 15, 07006 (2017).

[6] M. Janeček, F. Nový, P. Harcuba, J. Stráský, L. Trško, M. Mhaede, L. Wagner, Acta Phys. Pol. A 128, 497 (2015).

[7] M. Walczak, K. Drozd, M. Szala, J. Caban, Chiang Mai, J. Sci. 46, 766 (2019).

[8] X. Huang, I. Etsion, T. Shao, Wear 338, 54 (2015).

[9] B. Kilınc, O. Cegil, S. Sen, U. Sen, Acta Phys. Pol. A 125, 362 (2014).

[10] J. Rao, A. Sharma, T. Rose, Coatings 8, 12 (2017).

[11] H. Çalışkan, M. Küçükköse, Int. J. Refract. Met. Hard Mater. 50, 304 (2015).

[12] F. Yıldız, A. Alsaran, A. Çelik, İ. Efeoğlu, Surf. Eng. 26, 578 (2010).

[13] G.S. Fox-Rabinovich, A.I. Kovalev, M.H. Aguirre, B.D. Beake, K. Yamamoto, S.C. Veldhuis, J.L. Endrino, D.L. Wainstein, A.Y. Rashkovskiy, Surf. Coat. Technol. 204, 489 (2009).

[14] H. Çalışkan, A. Karaoğlanlı, Acta Phys. Pol. A $\mathbf{1 2 5}$, 456 (2014).

[15] B. Ahmad, S.O.V.D. Veen, M.E. Fitzpatrick, H. Guo, Addit. Manuf. 22, 571 (2018).

[16] M. Benedetti, E. Torresani, M. Leoni, V. Fontanari, M. Bandini, C. Pederzolli, C. Potrich, J. Mech. Behav. Biomed. Mater. 71, 295 (2017).

[17] N. Kazantseva, P. Krakhmalev, I. Yadroitsev, A. Fefelov, A. Merkushev, M. Ilyinikh, N. Vinogradova, I. Ezhov, T. Kurennykh, Mater. Lett. 209, 311 (2017).

[18] Q.X. Fan, T.G. Wang, Y.M. Liu, Z.H. Wu, T. Zhang, T. Li, Z.B. Yang, Acta Metall. Sin. Engl. Lett. 29, 1119 (2016).

[19] F. Cai, X. Huang, Q. Yang, Wear 324, 27 (2015).

[20] W.C. Oliver, G.M. Pharr, J. Mater. Res. 7, 1564 (1992).

[21] W.J. Shen, M.H. Tsai, J.W. Yeh, Coatings 5, 312 (2015).

[22] N. Vidakis, A. Antoniadis, N. Bilalis, J. Mater. Process. Technol. 143, 481 (2003).

[23] K. Pasierbiewicz, A. Rzepecka, M. Walczak, ITM Web Conf. 1506006 (2017).

[24] M. Łępicka, M. Grądzka-Dahlke, D. Pieniak, K. Pasierbiewicz, A. Niewczas, Wear 382, 62 (2017).

[25] J.K. Chen, C.L. Chang, Y.N. Shieh, K.J. Tsai, B.H. Tsai, Procedia Eng. 36, 335 (2012). 